\title{
Robustness of the nonequilibrium entropy related to the Maxwell-Cattaneo heat equation
}

\author{
F. X. Alvarez ${ }^{*}$ and J. Casas-Vázquez ${ }^{\dagger}$ \\ Departament de Física, Universitat Autònoma de Barcelona, 08193 Bellaterra, Catalonia, Spain \\ D. $\mathrm{Jou}^{\ddagger}$ \\ Departament de Física, Universitat Autònoma de Barcelona, 08193 Bellaterra, Catalonia, Spain \\ and Institut d'Estudis Catalans, Carme 47, Barcelona 08001, Catalonia, Spain
}

(Received 13 December 2007; published 10 March 2008)

\begin{abstract}
The connection between the Maxwell-Cattaneo heat transport equation and a nonequilibrium entropy is examined through four different thermodynamic approaches, and it is shown that all of them lead to the same form of the nonequilibrium entropy. Furthermore, it is seen that this form is also consistent with three microscopic formalisms. This robustness underlines the consistency and relevance of the entropy.
\end{abstract}

DOI: $10.1103 /$ PhysRevE.77.031110

PACS number(s): 05.70.Ln, 66.25.+g, 65.40.gd

\section{INTRODUCTION}

Nonequilibrium thermodynamics is an open and active field of research where a relatively high number of different approaches coexist [1-13]. The differences between them have been pointed out on several occasions, but their confluences have not yet received sufficient emphasis, maybe because of the difficulties caused by the choice of different variables, different formulations of the second law, and attention to different kinds of physical systems and different problems. The aim of this paper is to emphasize that different formalisms lead to the same nonequilibrium entropy in some circumstances. This confluence of different approaches deserves to be outlined because it gives more consistency to the final common result. Since entropy outside equilibrium is a particularly debated topic, expressions for nonequilibrium entropies are especially worthy of attention.

The present analysis is focused on a concrete situation, arising in heat transport with some memory effects. The Maxwell-Cattaneo equation is a well-known extension of the Fourier heat transport equation incorporating a relaxational term, which leads to a finite velocity of propagation of heat pulses $[1,7,8,14-18]$. It reads

$$
\tau \dot{\boldsymbol{q}}+\boldsymbol{q}=-\lambda \boldsymbol{\nabla} T,
$$

where $\lambda$ is the thermal conductivity and $\tau$ the relaxation time of the heat flux. The speed of the heat pulses is given by $v^{2}=\lambda / \rho c_{v} \tau$, with $\rho$ the mass density and $c_{v}$ the heat capacity per unit mass at constant volume; when $\tau$ tends to zero, the velocity of the heat pulses diverges, whereas (1) reduces to the classical Fourier law.

Despite its simplicity, Eq. (1) has stimulated much research in nonequilibrium thermodynamics, in order to obtain theoretical models in which this equation becomes compatible with the second law of thermodynamics $[1,5,7,8,14,19]$. This is necessary because Eq. (1) is not compatible with the local-equilibrium formulation of the second law, as it leads to

\footnotetext{
*Xavier.Alvarez@uab.es

†Jose.Casas@uab.es

”David.Jou@uab.es
}

negative entropy production in some situations. Here, we want to stress a result that is common to all the mentioned theoretical approaches, when expressed in terms of the same variables, namely, the consistency between (1) and the following form of the entropy per unit volume:

$$
s(u, \boldsymbol{q})=s_{\mathrm{eq}}(u)-\frac{\tau}{2 \lambda T^{2}} \boldsymbol{q} \cdot \boldsymbol{q},
$$

where $s_{\text {eq }}(u)$ is the local-equilibrium entropy per unit volume, a function of the internal energy per unit volume, $u$ (here, we focus our attention on heat transfer in incompressible systems and neglect other possible classical variables of the entropy).

This is a nonequilibrium entropy, because of the contribution of the second term on the right-hand side, which depends on the heat flux and vanishes at equilibrium. Note the close relation between this term in (2) and the relaxational term in (1): both of them vanish when $\tau$ tends to zero.

However, the robustness of the connection between (1) and (2) has not been appreciated enough up to now, because it is usually credited only to the theory known as extended irreversible thermodynamics (EIT) $[7,8,14,16,19]$. Here we will show, however, that this connection has also been established in other approaches, in the linear domain, with different choices of variables and different methods of exploitation of the second law. This was not sufficiently realized up to now because the corresponding entropies were always expressed in terms of different variables and without using an explicit physical identification of the coefficient of the nonequilibrium term. To be more faithful to the original developments and to better understand the difficulties in realizing the mentioned relation, we will keep the notation of each theory rather than unifying the different notations a priori. We think that this robustness of relation (2) to different methods of derivation has much interest, because it illustrates an explicit form of a nonequilibrium entropy common to different approaches.

\section{EXTENDED IRREVERSIBLE THERMODYNAMICS}

In EIT, a thermodynamic theory that incorporates the fluxes in the set of independent thermodynamic variables 
$[1,7,14,19]$, one starts from an entropy of the form $s(u, \boldsymbol{q})$. In fact, more general forms are used, incorporating more variables, either classical, such as the volume and the composition of the system, and nonequilibrium ones, such as diffusion flux, electric current, or viscous pressure. Here, we will focus our attention on heat conduction. The heat flux is considered as an independent variable because (1) is an evolution equation for $\boldsymbol{q}$ which requires initial conditions for $\boldsymbol{q}$ to be solved. In second order in the heat flux, it is assumed that

$$
d s=\theta^{-1} d u-\alpha \boldsymbol{q} \cdot d \boldsymbol{q},
$$

where $\theta$ is the temperature, $s$ the entropy, $u$ the internal energy per unit volume, and $\alpha$ a coefficient which should be identified in physical terms. In fact, the temperature $\theta$ is assumed to be not exactly the local equilibrium temperature itself, but to contain a second-order contribution in the heat flux stated by the integrability condition of (3), namely, $\partial \theta^{-1} / \partial \boldsymbol{q}=-(\partial \alpha / \partial u) \boldsymbol{q}$, which leads, after integration, to

$$
\theta^{-1}=T^{-1}-\frac{1}{2}\left(\frac{\partial \alpha}{\partial u}\right) \boldsymbol{q} \cdot \boldsymbol{q} .
$$

If we are interested only in first-order corrections we can approximate the temperature by its local equilibrium temperature value $T$.

To simplify the presentation and concentrate our attention on the central point, we will assume incompressible systems. The energy balance equation has the form

$$
\dot{u}=-\boldsymbol{\nabla} \cdot \boldsymbol{q} .
$$

Then the rate of change of $s$ is

$$
\dot{s}=\theta^{-1} \dot{u}-\alpha \boldsymbol{q} \cdot \dot{\boldsymbol{q}}=-\boldsymbol{\nabla} \cdot\left(\theta^{-1} \boldsymbol{q}\right)+\boldsymbol{q} \cdot\left(\boldsymbol{\nabla} \theta^{-1}-\alpha \dot{\boldsymbol{q}}\right) .
$$

The first term in the right-hand side is the divergence of the entropy flux, namely, the exchange of entropy with the surroundings, and the second term is the entropy production. Since we want to find an evolution equation for $\boldsymbol{q}$, compatible with the positive definite character of the entropy production, we write

$$
\boldsymbol{\nabla} \theta^{-1}-\alpha \dot{\boldsymbol{q}}=\mu \boldsymbol{q},
$$

with $\mu$ a positive coefficient. Comparison of (7) with (1) leads to $\mu=\left(\lambda T^{2}\right)^{-1}$ and $\alpha=\left(\tau / \lambda T^{2}\right)$. Introducing the latter identification in (3) we have for the entropy

$$
d s=\theta^{-1} d u-\frac{\tau}{\lambda T^{2}} \boldsymbol{q} \cdot d \boldsymbol{q} .
$$

Here we have used the notation $\theta$ for the nonequilibrium temperature; relation (4) defines a nonequilibrium thermodynamic temperature which has been compared with other proposals for effective nonequilibrium temperatures [20].

Integration of (8) leads to (2), and relation (4) becomes

$$
\theta^{-1}=T^{-1}-\frac{1}{2} \frac{\partial}{\partial u}\left(\frac{\tau}{\lambda T^{2}}\right) \boldsymbol{q} \cdot \boldsymbol{q} .
$$

Now we will compare results (8) and (9) with those obtained in other approaches.

\section{INTERNAL VARIABLES}

The preceding derivation of the connection between (1) and (2) is almost immediate. The choice of $\boldsymbol{q}$ as independent variable is not shared by all theories but is typical of EIT which analyzes the minimum ingredients for the thermodynamic consistency of (1). One would like to understand (1) from some structural details of the system, rather than deriving it in a direct but less microscopically suggestive way. A possibility for deriving (1) on thermodynamical grounds is to assume the existence of a vectorial internal variable $\boldsymbol{\xi}$ characterizing some internal features of the system, to be identified later on $[4,10]$. Such an approach yields an equation that is more general than (1), but that, when specialized to (1), yields again the entropy (2).

To see this, we write the time derivative of $s(u, \xi)$ as

$$
\dot{s}=\theta^{-1} \dot{u}-\alpha^{\prime} \boldsymbol{\xi} \cdot \dot{\boldsymbol{\xi}},
$$

where the coefficient $\alpha^{\prime}$ plays a similar role as $\alpha$ in Eq. (3) of the previous section.

Taking into account the energy balance equation (5), (10) is written as

$$
\dot{s}=-\boldsymbol{\nabla} \cdot\left(\theta^{-1} \boldsymbol{q}\right)+\boldsymbol{q} \cdot \boldsymbol{\nabla} \theta^{-1}-\alpha^{\prime} \boldsymbol{\xi} \cdot \dot{\boldsymbol{\xi}} .
$$

As in Eq. (6), the first term represents the divergence of the entropy flux and the two last ones are the entropy production, which has a different structure than in (6). For the moment we will neglect nonlinear contributions of $\boldsymbol{q}$ to $\theta$, and we approximate it by $T$.

By following the usual procedure in classical irreversible thermodynamics [1,2,21], we will express $\boldsymbol{q}$ and $\dot{\xi}$ in terms of $\boldsymbol{\nabla} T^{-1}$ and $-\alpha^{\prime} \boldsymbol{\xi}$ in linear approximation as

$$
\begin{aligned}
& \boldsymbol{q}=L_{11} \nabla T^{-1}-L_{12} \alpha^{\prime} \boldsymbol{\xi}, \\
& \dot{\boldsymbol{\xi}}=L_{21} \nabla T^{-1}-L_{22} \alpha^{\prime} \boldsymbol{\xi},
\end{aligned}
$$

with $L_{i j}$ being phenomenological coefficients. We assume that $\boldsymbol{\xi}$ is odd under time reversal, a feature that will be discussed below. This leads, according to the Onsager-Casimir reciprocal relations, to $L_{12}=-L_{21}$ because $\boldsymbol{\nabla} T^{-1}$ is even and $\boldsymbol{\xi}$ odd, and the Onsager-Casimir relations state that $L_{j i}=\epsilon_{i} \epsilon_{j} L_{i j}$, with $\epsilon_{i}$ and $\epsilon_{j}$ being +1 or -1 if the corresponding variable is even or odd with respect to time reversal. The positiveness of the entropy production requires $L_{11}>0, L_{22}>0, L_{11} L_{22}$ $-L_{12} L_{21} \geq 0$. Combining (12) and (13) yields

$$
\dot{\boldsymbol{q}}+L_{22} \boldsymbol{q}=-\frac{1}{T^{2}} \alpha^{\prime}\left(L_{11} L_{22}-L_{12} L_{21}\right) \boldsymbol{\nabla} T-\frac{L_{11}}{T^{2}} \boldsymbol{\nabla} \dot{T} .
$$

The last term is not found in (1). An equation of the form (14), or in more explicit terms

$$
\tau_{1} \dot{\boldsymbol{q}}+\boldsymbol{q}=-\lambda \boldsymbol{\nabla} T-\lambda \tau_{2} \boldsymbol{\nabla} \dot{T},
$$

has been proposed by Tzou [17] in the double-lag method, $\tau_{1}$ and $\tau_{2}$ being relaxation terms; in rheology, an equation analogous to (15) but relating the viscous pressure tensor with the symmetric part of the velocity gradient is known as 
Jeffrey's equation $[1,2]$. Such an equation does not lead to hyperbolic equations for heat transport, and it leads to an infinite velocity for heat pulses. In EIT, an equation of the form (15) is obtained by taking as the independent variable not only $\boldsymbol{q}$ but also the flux of the heat flux, as we will discuss below $[1,7]$.

If we want to obtain (1) from (14), one must identify $L_{11}=0$, and it follows that $L_{22} \alpha^{\prime}=\tau^{-1}$ and $L_{12}^{2} \alpha^{\prime}=T^{2} \lambda / \tau$. But if $L_{11}=0$, then, according to (12), $\boldsymbol{\xi}=-\left(L_{12} \alpha^{\prime}\right)^{-1} \boldsymbol{q}$. In fact, $\alpha^{\prime}$ may be taken as 1 , by normalizing the definition of $\boldsymbol{\xi}$, and (10) may be rewritten in the form

$$
d s=T^{-1} d u-\frac{1}{L_{12}^{2}} \boldsymbol{q} \cdot d \boldsymbol{q},
$$

which is the same expression as (8) because of the identification of $L_{12}^{2}$ in terms of $\tau$ and $\lambda$ presented above and leads, under the same conditions, to (2). The importance of considering $\boldsymbol{\xi}$ as an odd variable with respect to time reversal may be realized a posteriori. Indeed, to eliminate the last term in (14) one must take $L_{11}=0$. Furthermore, one needs to identify $-L_{12} L_{21} / T^{2}=\lambda \geq 0$; therefore, it is necessary that $L_{12}$ and $L_{21}$ have different signs, which, by virtue of the OnsagerCasimir reciprocal relations requires that $\boldsymbol{\xi}$ has opposite parity to $\boldsymbol{\nabla} T^{-1}$, i.e., that it is an odd variable. This is consistent with the identification $\boldsymbol{\xi}=-L_{12}^{-1} \boldsymbol{q}$ because $\boldsymbol{q}$ is an odd variable. In different systems, the physical meaning of $\boldsymbol{\xi}$ may be different, and also the meaning of $L_{12}$. For instance, in ideal gases $\boldsymbol{\xi}$ may be related to the energy flux transported by moving molecules, whereas in superfluids it may be related to the relative motion between normal and superfluid components [22].

In going from (10) to (16) we have assumed that $\left(\tau / \lambda T^{2}\right)^{1 / 2}$ is constant. However, (16) is also valid in the general case, provided $T$ is not identified with the local equilibrium temperature. Indeed, in strict terms, combining (10), the identification of $\boldsymbol{\xi}$ in terms of $\boldsymbol{q}$, and the identification of $L_{12}$ as $\left(\lambda T^{2} / \tau\right)^{1 / 2}$, we should have written

$$
d s=T^{-1} d u-\left(\frac{\tau}{\lambda T^{2}}\right)^{1 / 2} \boldsymbol{q} \cdot d\left[\left(\frac{\tau}{\lambda T^{2}}\right)^{1 / 2} \boldsymbol{q}\right]
$$

When the differential in the second term is developed we obtain

$$
d s=\theta^{-1} d u-\left(\frac{\tau}{\lambda T^{2}}\right) \boldsymbol{q} \cdot d \boldsymbol{q},
$$

with $\theta$ given by

$$
\theta^{-1} \equiv T^{-1}-\frac{1}{2} \frac{\partial}{\partial u}\left(\frac{\tau}{\lambda T^{2}}\right) \boldsymbol{q}^{2}
$$

which is the same nonequilibrium thermodynamic temperature as in (9). Thus, the use of a vectorial internal variable odd with respect to time inversion allows one to obtain the Maxwell-Cattaneo relation (1) provided the entropy takes the form (2).

\section{RATIONAL EXTENDED THERMODYNAMICS}

In the two previous sections, the second law has been exploited along the lines of classical irreversible thermodynamics, namely, the Onsager linear relation between fluxes and forces. One may exploit the second law along the lines of rational thermodynamics $[1,12,13]$. In this section we do so by using the Liu technique in rational extended thermodynamics, selecting $u$ and $\boldsymbol{q}$ as variables; we will look for an evolution equation for $\boldsymbol{q}$ obeying the restrictions of the second law $[7,8]$.

Now, the second law is expressed as

$$
\dot{s}+\nabla \cdot J^{s} \geq 0
$$

with $\boldsymbol{J}^{s}$ the entropy flux. This equation is supplemented by the energy balance equation, and the evolution equation of $\boldsymbol{q}$, which is assumed to be of the general form

$$
\dot{\boldsymbol{q}}=-\boldsymbol{\nabla} \cdot \boldsymbol{Q}+\sigma_{q},
$$

where $Q$ is the heat flux and $\sigma_{q}$ is the source term. Note that this form is simply the general form of the evolution equations, incorporating the divergence of a flux-i.e., the exchange with the surroundings of the system-and a source term. To have a concrete equation, $\boldsymbol{q}$ and $\sigma_{q}$ must be specified. This is what we will do below.

To take into account the constraints of the evolution equations (5) and (21) besides the constraints of the second law (20), in Liu's method one incorporates these dynamical constraints by means of Lagrange multipliers, as

$$
\dot{s}+\boldsymbol{\nabla} \cdot \boldsymbol{J}^{s}-\Lambda_{0}(\dot{u}+\boldsymbol{\nabla} \cdot \boldsymbol{q})-\Lambda_{1} \cdot\left(\dot{\boldsymbol{q}}+\boldsymbol{\nabla} \cdot \boldsymbol{Q}-\sigma^{q}\right) \geq 0 .
$$

Note that, for the moment, $s$ and $\boldsymbol{J}^{s}$ have not been assigned an explicit form, but they will be found consistently throughout the development. This is a difference with respect to the classical theory of nonequilibrium thermodynamics, in which $s$ is taken as the local equilibrium entropy and $\boldsymbol{J}^{s}$ is identified a priori with $T^{-1} \boldsymbol{q}$. In EIT and internal variable theory, $s$ is not fully identified a priori, but some coefficients in the differential of the entropy must be identified in consistency with the evolution equations.

Condition (22) leads to

$$
\begin{gathered}
\left(\frac{\partial s}{\partial u}-\Lambda_{0}\right) \dot{u}+\left(\frac{\partial s}{\partial \boldsymbol{q}}-\boldsymbol{\Lambda}_{1}\right) \cdot \dot{\boldsymbol{q}}+\frac{\partial \boldsymbol{J}^{s}}{\partial u} \cdot \boldsymbol{\nabla} u+\left(\frac{\partial \boldsymbol{J}^{s}}{\partial \boldsymbol{q}}-\Lambda_{0} \mathbf{U}\right): \nabla \boldsymbol{q} \\
-\boldsymbol{\Lambda}_{1} \cdot(\boldsymbol{\nabla} \cdot \boldsymbol{Q})+\boldsymbol{\Lambda}_{1} \cdot \boldsymbol{\sigma}^{q} \geq 0 .
\end{gathered}
$$

This expression must be positive for any values of $\dot{u}, \dot{\boldsymbol{q}}, \boldsymbol{\nabla} u$, and $\boldsymbol{\nabla} \boldsymbol{q}$; this implies that the coefficients of these quantities in (23) must vanish because $\dot{u}, \boldsymbol{q}, \boldsymbol{\nabla} u$, and $\boldsymbol{\nabla} \boldsymbol{q}$ may take arbitrary positive or negative values. Thus it follows that $\partial s / \partial u=\Lambda_{0}, \partial s / \partial \boldsymbol{q}=\boldsymbol{\Lambda}_{1}$, and $\boldsymbol{J}^{s}=\Lambda_{0} \boldsymbol{q}$. According to these conclusions one may write

$$
\begin{gathered}
d s=\Lambda_{0} d u+\Lambda_{1} \cdot d \boldsymbol{q}, \\
\boldsymbol{J}^{s}=\Lambda_{0} \boldsymbol{q} .
\end{gathered}
$$

From a physical point of view, these expressions are not very illustrative, unless $\Lambda_{0}$ and $\boldsymbol{\Lambda}_{1}$ are identified in physical terms 
and related to measurable quantities. For $\boldsymbol{q}=\mathbf{0}$, (24) should reduce to the classical Gibbs equation, which leads to the identification $\Lambda_{0}=T^{-1}$ near equilibrium; this is also consistent, when introduced in (25), with the classical form of the entropy flux. A main point is to identify $\boldsymbol{\Lambda}_{1}$ when $\boldsymbol{q}$ is not zero. Furthermore, the integrability condition of (24) is

$$
\frac{\partial \Lambda_{0}}{\partial \boldsymbol{q}}=\frac{\partial \boldsymbol{\Lambda}_{1}}{\partial u},
$$

which leads to

$$
\Lambda_{0}(u, \boldsymbol{q})=T^{-1}+\int \frac{\partial \boldsymbol{\Lambda}_{1}}{\partial u} \cdot d \boldsymbol{q} .
$$

One may identify $\Lambda_{0} \equiv \theta^{-1}$ as a nonequilibrium temperature, as in previous sections.

The expression for $\Lambda_{1}$ will be related to the evolution equation for $\boldsymbol{q}$, given by (21). Now, we assume $\boldsymbol{Q}=a(u) \boldsymbol{U}$ and $\sigma^{q}=-\tau^{-1} \boldsymbol{q}$ as a particular and simplest possibility, which does not exclude other more general possibilities. In this case the residual terms in (23) may be written as

$$
\left(\frac{\partial J^{s}}{\partial u}-\Lambda_{1} \frac{\partial a}{\partial u}\right) \cdot \nabla u-\tau^{-1} \Lambda_{1} \cdot \boldsymbol{q} \geq 0 .
$$

Since $\partial \boldsymbol{J}^{s} / \partial u=\left(\partial \theta^{-1} / \partial u\right) \boldsymbol{q}$, it follows from the first term in parentheses in (28) that

$$
\boldsymbol{\Lambda}_{1}=\frac{1}{\left(\partial a / \partial \theta^{-1}\right)} \boldsymbol{q} .
$$

This shows explicitly the connection between $\Lambda_{1}$ and the evolution equation (21). To be totally explicit, the function $a(u)$ must yet be identified.

Introducing into (21) the forms of $\boldsymbol{q}$ and $\sigma^{q}$ postulated above, we obtain

$$
\dot{\boldsymbol{q}}=-\boldsymbol{\nabla} a-\tau^{-1} \boldsymbol{q} .
$$

Comparing (30) to (1), and writing $\boldsymbol{\nabla} a=(\partial a / \partial \theta) \boldsymbol{\nabla} \theta$, we may identify $(\partial a / \partial \theta)=\lambda / \tau$. From here and (29) it follows that

$$
\boldsymbol{\Lambda}_{1}=\frac{\partial \theta^{-1}}{\partial a} \boldsymbol{q}=-\frac{1}{\theta^{2}}\left(\frac{\partial \theta}{\partial a}\right) \boldsymbol{q}=-\frac{\tau}{\lambda \theta^{2}} \boldsymbol{q} .
$$

Then the Gibbs equation may be written as

$$
d s=\Lambda_{0} d u+\Lambda_{1} \cdot \boldsymbol{q}=\theta^{-1} d u-\frac{\tau}{\lambda \theta^{2}} \boldsymbol{q} \cdot d \boldsymbol{q},
$$

which has, again, the form (6) or (16), and leads to (2), under the assumption of the integrability conditions of (32). This condition is (26) and leads to the same form as (9) for the nonequilibrium temperature $\theta$.

Unfortunately, in the standard presentations of rational extended thermodynamics [8], the explicit identification of $\boldsymbol{\Lambda}_{1}$ is usually not given. This makes it difficult to realize that the entropy (2) is recovered in this case.

\section{INTERNAL VARIABLES AND RATIONAL THERMODYNAMICS}

The assumption that some internal variables must be included in the description of the system does not imply by itself which kind of interpretation and of exploitation of the second law must be used. In Sec. III, we have used the Onsager approach. Here, we will use instead the framework of rational thermodynamics.

In particular, we will follow a paper by Cimmelli and Frischmuth based on a particular interpretation of the internal variable [23]. Following a model proposed by Koscinsky they assume that the heat flux is given by

$$
q=-\nabla \beta,
$$

where $\beta$ is a generalized dynamical temperature which in steady state becomes equal to the temperature $\theta$, according to an evolution equation of the generic form

$$
\dot{\beta}=f(\theta, \beta),
$$

and they denote $\boldsymbol{p} \equiv \boldsymbol{\nabla} \beta$. They assume that $\beta$ does not enter the constitutive equations explicitly, and that $\boldsymbol{p} \equiv \boldsymbol{\nabla} \beta$ can be regarded simply as an additional internal variable related to the heat flux. They postulate for $\boldsymbol{p}$ the equation

$$
\frac{\partial p}{\partial t}=-\frac{1}{\tau} \nabla \theta-\frac{1}{\sigma} p,
$$

where $\tau$ and $\sigma$ are positive quantities given by $\tau \equiv 1 / f_{1} \theta$ and $\sigma \equiv 1 / f_{1} \beta$. In the classical case, $\sigma=\tau$ and

$$
\dot{\beta}=\frac{\theta-\beta}{\tau} .
$$

The second law of thermodynamics is written as

$$
\psi_{, t}+\eta \theta_{, t}+\frac{1}{\theta} \boldsymbol{q} \cdot \boldsymbol{g} \leq 0,
$$

where $\boldsymbol{g} \equiv \boldsymbol{\nabla} \theta, \eta$ is the specific entropy, and $\psi \equiv \epsilon-\theta \eta$ is the Helmholtz free energy. Cimmelli and Frischmuth postulate that $\boldsymbol{q}, \boldsymbol{\epsilon}$, and $\psi$ are functions of $\theta, \boldsymbol{g}$, and $\boldsymbol{p}$. The corresponding constitutive equations for $\boldsymbol{q}, \boldsymbol{\epsilon}$, and $\psi$ are then examined from the perspective of their compatibility with the second law (37) by using Liu's method of Lagrange multipliers [8].

Thus, they write

$$
\psi_{, t}+\eta \phi_{, t}+\frac{1}{\theta} q \boldsymbol{g}-\lambda_{\epsilon}\left(\epsilon_{, t}+\boldsymbol{p}_{, x}\right)-\lambda_{p}\left(\boldsymbol{p}_{, t}+\frac{1}{\tau} \boldsymbol{g}+\frac{1}{\sigma} \boldsymbol{p}\right) \leq 0 .
$$

This leads, after a thorough application of the method of Lagrange multipliers, analogous to that shown in the previous section, to the necessary and sufficient conditions

$$
\lambda_{\epsilon}=0, \quad \eta=-\frac{\partial \psi}{\partial \theta}, \quad \frac{\partial \psi}{\partial \boldsymbol{p}}=\tau \lambda_{p}-\frac{\tau \boldsymbol{q}}{\theta},
$$

and the residual inequality

$$
\left(\lambda_{p}+\frac{q}{\theta}\right) \cdot p \leq 0
$$

Afterward, Cimmelli and Frischmuth formulate the hypothesis that 


$$
\boldsymbol{q}=-k(\theta, p) \boldsymbol{p},
$$

with $k(\theta, p)$ being a dynamical heat conductivity. This leads for the entropy inequality (40) to

$$
\lambda_{p} p-\frac{k}{\theta} p^{2} \leq 0
$$

It follows that $\lambda_{p}=0$ and $k>0$. To investigate the consequences of the hypothesis (41) they assume

$$
\begin{gathered}
\psi(\theta, \boldsymbol{p})=\psi_{\mathrm{eq}}(\theta)+\frac{1}{2} \psi_{2}(\theta) p^{2}, \\
\psi_{2}(\theta)=\frac{\tau}{\theta} .
\end{gathered}
$$

Then, by the restrictions (40) and (39), they find that

$$
\eta=-\frac{\partial \psi_{\mathrm{eq}}}{\partial \theta}-\frac{1}{2} \frac{\partial \psi_{2}}{\partial \theta} p^{2}=\eta_{\mathrm{eq}}-\frac{1}{2} \frac{\tau k}{\theta^{2}} p^{2}=\eta_{\mathrm{eq}}-\frac{1}{2} \frac{\tau}{k \theta^{2}} q^{2} .
$$

The result (45) is valid for $\tau k / \theta^{2}=$ const. In general, the entropy is

$$
\eta(\theta, \boldsymbol{q})=\eta_{\mathrm{eq}}(\theta)-\frac{1}{2} \frac{d}{d \theta}\left(\frac{\tau k}{\theta}\right) \frac{q^{2}}{\lambda^{2}} .
$$

To make contact with our previous results it must be recalled that $\eta_{\mathrm{eq}}$ should be referred to $\eta_{\mathrm{eq}}(\epsilon)$. According to Cimmelli and Frischmuth [23], one has

$$
\boldsymbol{\epsilon}=\epsilon_{\mathrm{eq}}+\frac{1}{2}\left[\frac{\tau k}{\theta}-\frac{1}{2} \theta \frac{\partial}{\partial}\left(\frac{\tau k}{\theta}\right)\right] \frac{q^{2}}{k^{2}},
$$

whereas in (46) $\eta_{\mathrm{eq}}$ is expressed in terms of $\theta$ corresponding to $\epsilon$. Thus, in (46) we should rewrite $\eta_{\mathrm{eq}}(\theta)$ in terms of $\eta\left(\epsilon_{\mathrm{eq}}\right)$. We have, from (47),

$$
\eta_{\mathrm{eq}}\left(\epsilon_{\mathrm{eq}}\right)=\eta_{\mathrm{eq}}\left\{\epsilon-\frac{1}{2}\left[\frac{\tau k}{\theta}-\frac{1}{2} \frac{d}{d \theta}\left(\frac{\tau k}{\theta}\right)\right] \frac{q^{2}}{k^{2}}\right\} .
$$

Expanding this expression up to second order in $q$ and recalling that $\partial \eta_{\mathrm{eq}} / \partial \epsilon=1 / \theta,(48)$ yields

$$
\eta_{\mathrm{eq}}\left(\epsilon_{\mathrm{eq}}\right)=\eta_{\mathrm{eq}}(\epsilon)-\frac{1}{2}\left[\frac{\tau k}{\theta^{2}}-\frac{1}{2} \frac{d}{d \theta}\left(\frac{\tau k}{\theta}\right)\right] \frac{q^{2}}{k^{2}} .
$$

When this is introduced into (46) we have

$$
\eta(\epsilon)=\eta_{\mathrm{eq}}(\epsilon)-\frac{1}{2} \frac{\tau}{\theta^{2} k} q^{2}
$$

This has the same form as the entropy (2), if one uses the symbol $s$ instead of $\eta$ to denote the entropy.

Note that to have the same result as in (2) it is necessary to be especially careful about the local equilibrium reference state, making sure that it is the same as in (2). Indeed, (46) seems rather different from (2), and one realizes the close connection only after realizing that in (2) entropies at the same internal energy are compared.

\section{MICROSCOPIC DERIVATIONS OF (2)}

The form (2) of the entropy, related to the form (1) for the evolution of $\boldsymbol{q}$, is not only obtained in different macroscopic formalisms but is also supported from several microscopic theories: (a) Grad's development of kinetic theory [24,25], (b) the Chapman-Enskog development of kinetic theory $[24,26]$, and (c) fluctuation expressions.

In kinetic theory for dilute gases, the entropy is given by the Boltzmann expression

$$
s(\boldsymbol{r}, t)=-k_{B} \int f(\boldsymbol{r}, \boldsymbol{c}, t) \ln f(\boldsymbol{r}, \boldsymbol{c}, t) d \boldsymbol{c},
$$

with $k_{B}$ the Boltzmann constant, $\boldsymbol{c}$ the peculiar molecular velocity, i.e., the velocity of the molecules with respect to the barycentric velocity of the system, and $f(\boldsymbol{r}, \boldsymbol{c}, t)$ the probability distribution function for the molecular velocity. At equilibrium $f$ is given by the well-known Maxwell-Boltzmann expression, which will be denoted as $f_{\text {eq }}(\boldsymbol{c})$. Near equilibrium, it is usual to write

$$
f=f_{\mathrm{eq}}\left(1+\Phi_{1}+\Phi_{2}+\cdots\right)
$$

where $\Phi_{i}$ are suitable nonequilibrium contributions, which must satisfy some side conditions in order that (51) refers to a given density, energy, and velocity. Introduction of (52) into (51) leads, up to second order, to $[1,8,24,25]$

$$
s=s_{\text {eq }}-\frac{k_{B}}{2} \int f_{\text {eq }} \Phi_{1}^{2} d c .
$$

The second term on the right-hand side of (53) indicates the nonequilibrium contribution to the entropy.

Our aim is now to explore whether these nonequilibrium contributions have the same form as (2) for heat transport. We will use two different formalisms: (a) Grad's approach, where $\Phi_{i}$ is expressed in terms of $\boldsymbol{q}$, and (b) the ChapmanEnskog approach, where $\Phi_{i}$ is given in terms of $\boldsymbol{\nabla} T$. It will be seen that, up to second order, both formalisms lead to the entropy (2).

\section{A. Grad's approach}

In Grad's approach, the distribution function $f$ is expanded in terms of its several moments with respect to the velocity; the zeroth-order moment is the density, the firstorder moment is the barycentric velocity, and the trace of the second-order moment is the internal energy. The heat flux is given by

$$
\boldsymbol{q}=\int f \frac{1}{2} m c^{2} \boldsymbol{c} d \boldsymbol{c}
$$

and therefore it is a part of the third-order moments of $f$. For heat transport, Grad's approach takes the form [24,26]

$$
\Phi_{1}(\boldsymbol{r}, t)=\left(\boldsymbol{A}(\boldsymbol{r}, t) \cdot \boldsymbol{c}+\boldsymbol{B}(\boldsymbol{r}, t) \cdot \frac{1}{2} m c^{2} \boldsymbol{c}\right),
$$

where $\boldsymbol{A}$ and $\boldsymbol{B}$ are coefficients determined in terms of the barycentric velocity $\boldsymbol{v}$ and the heat flux $\boldsymbol{q}$. When this is done, the explicit form of $(55)$ is $[24,26]$ 


$$
\Phi_{1}=\frac{2 m}{5 p k_{B}^{2} T^{2}}\left(\frac{1}{2} m c^{2}-\frac{5}{2} k_{B} T\right) \boldsymbol{c} \cdot \boldsymbol{q} .
$$

When this expression is inserted into (53) and after performing a suitable integration we find

$$
s=s_{\mathrm{eq}}-\frac{m}{5 p k_{B} T^{2}} \boldsymbol{q} \cdot \boldsymbol{q} .
$$

This shows that a contribution of second order in $\boldsymbol{q}$ to the entropy is to be expected on microscopic grounds. However, we want more than a qualitative corroboration. Therefore, we must examine whether (57) has the same form as (2). To do that, we need an evolution for the heat flux. In Grad's approach, this is obtained by inserting (55) into Boltzmann's equation and integrating it over the velocity. In the absence of viscous pressure contributions, this leads to $[24,26]$

$$
\frac{3}{2 \rho \gamma} \dot{\boldsymbol{q}}=-\boldsymbol{q}-\frac{15 p k_{B}}{4 m \rho \gamma} \nabla T,
$$

where $\gamma$ is a function of $T$ and $p$ related to an integral of the collision term. This equation has the form of (2) provided one identifies

$$
\tau_{1} \equiv \frac{3}{2 \rho \gamma}, \quad \lambda \equiv \frac{15 p k_{B}}{4 m \rho \gamma}=\frac{5}{2} \frac{p k_{B}}{m} \tau_{1} .
$$

From the expression relating $\lambda$ and $\tau_{1}$, it is seen that $\tau_{1} / \lambda$ $=2 \mathrm{~m} / 5 \mathrm{p} k_{B}$ and therefore that (57) may be written as

$$
s=s_{\mathrm{eq}}-\frac{\tau_{1}}{2 \lambda T^{2}} \boldsymbol{q} \cdot \boldsymbol{q},
$$

which is precisely the form (2) of the entropy.

\section{B. Relaxation-time approximation}

In nonequilibrium steady states one may approximate $\boldsymbol{q}$ by its classical expression in terms of the temperature gradient, according to Fourier's law. Then, (2) becomes

$$
s=s_{\mathrm{eq}}-\frac{\tau_{1} \lambda}{2 T^{2}}(\nabla T) \cdot(\nabla T)
$$

This expression for the entropy is also obtained from a microscopic basis in the relaxation-time approximation in the Chapman-Enskog approach [25]. In such an approach, the nonequilibrium correction to the distribution function is expressed in terms of $\boldsymbol{\nabla} T$ instead of in terms of $\boldsymbol{q}$. In this case, one has

$$
\boldsymbol{\Phi}_{1}=-\frac{\tau_{1}}{k_{B} T^{2}}\left(\frac{1}{2} m c^{2}-\frac{5}{2} k_{B} T\right) c \cdot \nabla T .
$$

When introduced into the entropy (53) and after performing the integration we find that

$$
s=s_{\mathrm{eq}}-\frac{5}{4} \frac{p k_{B} \tau_{1}^{2}}{m T^{2}} \nabla T \cdot \nabla T .
$$

In view of the form (59) for $\lambda$ in terms of $\tau_{1}$, which is also found in the Chapman-Enskog approach, this may be rewritten just as (61).

\section{Fluctuation-dissipation expressions}

A third microscopic proof of the internal consistency of (1) and (2) may be obtained by studying the second moment of the fluctuations of the heat flux around equilibrium. This is more general than the two previous derivations in this section, because it is not restricted to classical diluted gases, but is valid for general systems. To do that, we use the wellknown Einstein formula for the probability of fluctuations, namely,

$$
\text { Prob } \sim \exp \left(\frac{1}{2} \frac{\delta^{2} s}{k_{B}}\right)
$$

where $\delta^{2} s$ is the second differential of the entropy. In fact, the Einstein formula is applicable to the fluctuations of extensive quantities; thus, instead of working with $\boldsymbol{q}$ itself, one should work with $V \boldsymbol{q}$, which is an extensive variable, as is given by

$$
V \boldsymbol{q}=\sum_{i=1}^{N} \frac{1}{2} m_{i} c_{i}^{2} \boldsymbol{c}_{i}
$$

where $N$ is the number of particles. By combining (2) and (64), and writing the volume explicitly, we have

$$
\text { Prob } \sim \exp \left(-\frac{1}{2} \frac{\tau}{\lambda V T^{2} k_{B}}(\delta V \boldsymbol{q})^{2}\right) .
$$

From here one obtains for the second moments of the fluctuations

$$
\left\langle\delta V \boldsymbol{q}_{i} \delta V \boldsymbol{q}_{j}\right\rangle=k_{B} \frac{\lambda V T^{2}}{\tau} \delta_{i j},
$$

with $\delta_{i j}$ being Kronecker's delta, and the angular brackets denoting an equilibrium average.

This expression is a particular case of the general GreenKubo expression for the thermal conductivity, according to which $[1,5]$

$$
\lambda \delta_{i j}=\frac{V}{k_{B} T^{2}} \int_{0}^{\infty}\left\langle\delta \boldsymbol{q}_{i} \delta \boldsymbol{q}_{j}\right\rangle d t
$$

If one uses (1) to describe the decay of the fluctuations $\delta q(t)$ near equilibrium one has $\delta \boldsymbol{q}_{i}(t)=\delta \boldsymbol{q}_{i}(0) \exp (-t / \tau)$. When this is introduced into (68) and the integration is performed, expression (67) is recovered. In other words, if one writes

$$
s=s_{\mathrm{eq}}-\frac{\alpha}{2} \boldsymbol{q} \cdot \boldsymbol{q},
$$

and one uses the Einstein expression for fluctuations, one finds

$$
\left\langle\delta \boldsymbol{q}_{i} \delta \boldsymbol{q}_{j}\right\rangle=\frac{k_{B}}{\alpha} \delta_{i j} .
$$

Then, consistency with (68) in the relaxation-time approximation would lead to $\alpha=\tau / \lambda T^{2}$, as in expression (2). Consistency between (1) and (2) is corroborated again. 


\section{COMMENTS}

In this paper we have shown that seven different methods, four of them macroscopic and three others microscopic, lead to the same expression for the nonequilibrium entropy (2) related to the Maxwell-Cattaneo equation (1). Such generality of $s(u, \boldsymbol{q})$ reinforces its role as a nonequilibrium entropy. Of course, the validity of such entropy is restricted to situations where Eq. (1) is valid, but it is of interest as a particular explicit illustration of a nonequilibrium entropy.

If quartic terms in $\boldsymbol{q}$ were added to the entropy, the corresponding evolution equation for the heat flux would not be the linear Maxwell-Cattaneo equation. Probably, the quarticorder approximation to the entropy would not be universal; for instance, approaches based on kinetic theory or on maximum entropy lead to the same result for the quadratic contributions of the heat flux to the entropy, but they lead to different coefficients for the quartic term [7]. In any case, the universality at the quadratic order is interesting by itself, as it covers a considerable phenomenology and it uncovers some degree of universality beyond the local-equilibrium hypothesis. This is valid not only for the heat flux, but also for other fluxes, such as, for instance, the momentum flux or viscous pressure tensor $\boldsymbol{P}^{v}$, in which case the quadratic nonequilibrium entropy per unit volume has the form $[7,19]$

$$
s\left(u, \boldsymbol{P}^{v}\right)=s_{\mathrm{eq}}(u)-\frac{\tau}{2 \nu T} \boldsymbol{P}^{v}: \boldsymbol{P}^{v},
$$

where $\nu$ is the shear viscosity and $\tau$ the viscoelastic relaxation time, appearing in the Maxwell viscoelasticity equation

$$
\tau \frac{d \boldsymbol{P}^{v}}{d t}+\boldsymbol{P}^{v}=-2 \nu \boldsymbol{V}
$$

with $\boldsymbol{V}$ the symmetric part of the velocity gradient.

Particular simplified models which are able to grasp some essential physics - in our case, a finite speed velocity of heat pulses-are useful as a basis for more exhaustive and detailed models. In this aspect, the entropy (2) plays a role analogous to that of the van der Waals equation in the analysis of real gases. The van der Waals equation is not an exact equation, and it does not lead to very accurate quantitative predictions, but it grasps the existence of a finite critical temperature. The entropy (2) is not an exact expression, and Eq. (1) does not lead to accurate expressions for heat wave speeds, but it is able to grasp a finite value for the speed of heat pulses. To improve the quantitative agreement, higherorder approaches are needed. In the van der Waals equation, they are related to higher-order powers of the density; in extended thermodynamics, they are related to the higherorder fluxes of the energy.

\section{ACKNOWLEDGMENTS}

We acknowledge the financial support of the Spanish Ministry of Education under Grant No. FIS2006-12296C02-01 and of Direcció General de Recerca of the Generalitat of Catalonia under Grant No. 2005 SGR00087.
[1] G. Lebon, D. Jou, and J. Casas-Vázquez, Understanding Nonequilibrium Thermodynamics (Springer, Berlin, 2008).

[2] H. C. Ottinger, Beyond Equilibrium Thermodynamics (John Wiley and Sons, New Jersey, 2005).

[3] W. Muschik, Aspects of Nonequilibrium Thermodynamics (World Scientific, Singapore, 1990).

[4] G. Maugin, The Thermodynamics of Nonlinear Irreversible Behaviours. An Introduction (World Scientific, Singapore, 1990).

[5] R. Luzzi, A. R. Vasconcellos, and J. Galvao Ramos, Predictive Statistical Mechanics: A Nonequilibrium Ensemble Formalism (Kluwer Academic, Dordrecht, 2002).

[6] B. C. Eu, Nonequilibrium Statistical Mechanics: Ensemble Method (Kluwer, Dordrecht, 1998).

[7] D. Jou, J. Casas-Vázquez, and G. Lebon, Extended Irreversible Thermodynamics, 3rd ed. (Springer, Berlin, 2001).

[8] I. Müller and T. Ruggeri, Rational Extended Thermodynamics (Springer, New York, 1997).

[9] K. Wilmansky, Thermomechanics of Continua (SpringerVerlag, Berlin, 1998).

[10] J. Verhas, Rheology and Thermodynamics (Kluwer, Dordrecht, 1997).

[11] A. N. Beris and B. J. Edwards, Thermodynamics of Flowing Systems: With Internal Microstructure (Oxford University Press, New York, 1994).

[12] C. Truesdell, Rational Thermodynamics (Springer-Verlag, New York, 1984).
[13] M. Silhavy, The Mechanics and Thermodynamics of Continuous Media (Springer-Verlag, Berlin, 1997).

[14] D. Jou, J. Casas-Vázquez, and G. Lebon, Rep. Prog. Phys. 51, 1105 (1988).

[15] D. D. Joseph and L. Preziosi, Rev. Mod. Phys. 61, 41 (1989).

[16] W. Dreyer and H. Struchtrup, Continuum Mech. Thermodyn. 5, 3 (1993).

[17] D. Y. Tzou, Macro-to-Microscale Heat Transfer: The Lagging Behavior, 3rd ed. (Taylor and Francis, New York, 1997).

[18] Z. Zhang, Nano/Microscale Heat Transfer (McGraw-Hill, New York, 2007).

[19] D. Jou, J. Casas-Vázquez, and M. Criado-Sancho, Thermodynamics of Fluids under Flow (Springer, Berlin, 2001).

[20] J. Casas-Vázquez and D. Jou, Rep. Prog. Phys. 66, 1937 (2003).

[21] S. R. de Groot and P. Mazur, Nonequilibrium Thermodynamics (North-Holland, Amsterdam, 1962).

[22] M. S. Mongiovi, Phys. Rev. B 48, 6276 (1993).

[23] V. A. Cimmelli and K. Frischmuth, Physica B 355, 147 (2004).

[24] S. Harris, An Introduction to the Theory of the Boltzmann Equation (Holt, Rinehart and Winston, New York, 1971).

[25] S. Chapman and T. G. Cowling, The Mathematical Theory of Nonuniform Gases (Cambridge University Press, Cambridge, UK, 1970).

[26] H. Grad, Principles of the Kinetic Theory of Gases, Handbuck der Physik Vol. 12 (Springer, Berlin, 1958). 